\title{
Elastic waves guided by a welded joint in a plate
}

\author{
By Zheng Fan and Mike J. S. Lowe \\ Department of Mechanical Engineering, Imperial College, London SWr 2AZ, UK
}

The inspection of large areas of complex structures is a growing interest for industry. An experimental observation on a large welded plate found that the weld can concentrate and guide the energy of a guided wave traveling along the direction of the weld. This is attractive for NDE since it offers the potential to quickly inspect for defects such as cracking or corrosion along long lengths of welds. In this paper, a two dimensional Semi Analytical Finite Element (SAFE) method is applied to provide a modal study of the elastic waves which are guided by the welded joint in a plate. This brings understanding to the compression wave which was previously observed in the experiment. However, during the study, a shear weld-guided mode, which is non-leaky and almost non-dispersive has also been discovered. Its characteristics are particularly attractive for NDE, so this is a significant new finding. The properties for both the compression and the shear mode are discussed and compared, and the physical reason for the energy trapping phenomena is then explained. Experiments have been undertaken to validate the existence of the shear weld-guided mode and the accuracy of the FE model, showing very good results.

Keywords: feature guiding, SAFE, shear mode

\section{INTRODUCTION}

Guided waves are interesting for large area inspections since they offer the potential for rapid screening from a single transducer position. Several successful applications have been made on one dimensional structures such as pipelines (Alleyne et al. 2001) and rails (Wilcox et al. 2003; Rose et al. 2002). Research work has also been carried out to study the possibility of applying the guided wave inspection to two dimensional plate-like structures such as storage tanks, pressure vessels and airframes (Lowe \& Diligent 2002; Lee \& Staszewski 2003; Wilcox et al. 2005), although this has resulted in little commercialization so far. This is mainly because in a two dimensional structure waves can propagate in an infinite number of directions from a single transducer position, and in each direction the energy of the spreading wave decays with the distance away from the source. Another challenge to inspect real plate-like structures is that there always exists some features such as welds and ribs, which may cause extra coherent noise which interferes with the inspection signal.

However, a recent experimental observation (Sargent 2006) on a large welded plate revealed that there existed 'weld-guided' modes which can propagate along the weld and concentrate the energy in and around the weld. Similar trapped modes have also been discovered by Postnova and Craster (2008) from an analytical calculation on a welded plate structure based on the long-wave theory. Fig. 1 schematically shows the propagation of a feature-guided wave on a welded plate from a pulse 
excitation. As can be seen from the figure, due to the geometry change part of the energy is trapped in and around the weld and propagates along the weld. This is very attractive for NDE as we know defects frequently occur preferentially in or near the weld, and the same is true for other geometric features, such as joints and stiffeners. Therefore, instead of seeing the features as a problem, it may be possible to exploit them as waveguides to focus the energy of the guided wave, which offers the potential to quickly inspect for defects such as corrosion along long lengths of features on plate-like structures.

In order to exploit this feature-guided wave, it is necessary to understand its nature and propagation characteristics. Juluri et al. (2007) performed a three dimensional time step finite element simulation on an idealized welded plate geometry, and demonstrated the existence of the compression weld-guided mode (similar to the Lamb S0 mode in the plate), which had been experimentally detected (Sargent 2006). However these simulations are very time consuming, and can only model the chosen frequency and wave mode, thus they are not sufficient to investigate the guided wave properties over ranges of parameters and feature geometries. In order to further understand how the guiding is affected by the geometry and frequency, it is therefore necessary to perform a modal study of the welded-plate, in order to fully predict the properties of the waves which are guided by the features. Such a model may then also create the possibility of finding other feature-guided modes which could be candidates for inspection but have not yet been discovered.

A Semi Analytical Finite Element (SAFE) method, which uses finite elements to represent the cross section of the waveguide, plus a harmonic description along the propagation direction, has become popular in recent years for the modal study of waveguides with irregular cross section, and has the potential for application to the case of feature guided waves. The SAFE method was first demonstrated in 1972 (Lagasse 1972), and has been used to calculate the properties of guided waves in railway lines (Gavrić 1995; Hayashi et al. 2003), beams (Wilcox et al. 2002) and stiffened plates (Orrenius \& Finnveden 1996). Recently the SAFE method has been developed by Castaings and Lowe (2008) to study leaky waves which propagate along an elastic waveguide with arbitrary cross section and radiate into a solid of infinite extent. They also presented a model of two large plates connected by a rectangular butt weld, and showed results for the compression weld-guided mode which had been found experimentally (Sargent 2006) and demonstrated by the time-step finite element simulation (Juluri et al. 2007).

In this paper, we apply the SAFE modeling on a geometry of a real welded plate and provide further investigation on the properties of feature guided waves and the physical reason for the energy trapping effect. Significantly, during the modal study, another interesting shear mode, which is similar to the SH0 mode in the plate, has been discovered. The particle displacement of this mode is perpendicular to plane of propagation and therefore it is expected to be more sensitive than the compression mode to the fatigue cracks that are typically aligned along the weld in the heat affected zone. In addition this shear mode has no leakage to the side plates and is almost non-dispersive, thus it is very interesting as a candidate mode for industrial inspection. Experiments have been set up to demonstrate the existence of this shear weld guided mode and the accuracy of the SAFE model, showing very good agreement.

This paper starts with a brief review of the SAFE theory in Sec.2 and follows 
with the modal study of the actual welded-plate in Sec.3. In this section, both compression and shear weld-guided waves are investigated and compared at a single frequency. Then the dispersion curves (frequency-velocity relationships), the possible attenuation by leakage of energy into the adjacent plates, and the mode shapes (distributions of stress and displacement across the section of the waveguide) are calculated and discussed for both modes. In Sec.4, the guiding phenomena are explained by comparing the mode shapes and the phase velocity of the weld cap and the adjacent plate. Finally, a validation experiment is introduced, and results of measurements are shown in Sec.5.

\section{THEORY}

Since the derivation of the SAFE theory in solids has been detailed in previous research work (Predoi et al. 2007), only the equations used in this paper will be briefly explained. The mathematical model of the SAFE method in solids is based on the three dimensional elasticity approach. Consequently, the displacement vector in the waveguide can be written:

$$
u_{i}\left(x_{1}, x_{2}, x_{3}, t\right)=U_{i}\left(x_{1}, x_{2}\right) e^{I\left(k x_{3}-\omega t\right)}, I=\sqrt{-1},
$$

in which $k$ is the wavenumber, $\omega=2 \pi f$ is the angular frequency, $f$ being the frequency, $t$ is the time variable and the subscript $i=1,2,3$. The harmonic guided waves are assumed to propagate along the $x_{3}$ direction. For general anisotropic material, the equation of dynamic equilibrium can be written in the following form of an eigenvalue problem:

$$
C_{i k j l} \frac{\partial^{2} U_{j}}{\partial x_{k} \partial x_{l}}+I\left(C_{i 3 j k}+C_{i k j 3}\right) \frac{\partial\left(k U_{j}\right)}{\partial x_{k}}-k C_{i 3 j 3}\left(k U_{j}\right)+\rho \omega^{2} \delta_{i j} U_{j}=0,
$$

with summation over the indices $j=1,2,3$ and $k, l=1,2$. The coefficients $C_{i j k l}$ are the stiffness moduli and $\delta_{i j}$ is the Kronecker symbol. In the commercial FEM code (COMSOL 2008) used in this study, the formalism for eigenvalue problems has the general expression:

$$
\nabla \cdot(c \nabla U+\alpha U-\gamma)-\beta \nabla U-a U+\lambda d_{a} U-\lambda^{2} e_{a} U=0
$$

in which all matrix coefficients are given by Predoi et al (2007).

The nature of the solution is thus to find eigenvalues of wavenumber $k$ for chosen values of angular frequency $\omega$. Each solution at a chosen frequency will reveal the wavenumbers of all of the possible modes at that frequency; then the full dispersion curve spectrum can be found by repeating the eigenvalue solutions over the desired range of frequencies.

\section{MODAL STUDY OF THE WELDED PLATE}

Compared to the conventional three dimensional time step finite element simulation, the two dimensional SAFE model has many advantages. With this method, only the cross section which is normal to the direction of the wave propagation has to be meshed by finite elements. A typical calculation (calculation of all the propagation wave numbers at one frequency) in our model presented here only takes 
approximately one minute on a Pentium 4 PC with 2 Gbyte memory, while it takes several hours to calculate one specific mode propagation at one frequency in the 3D time step FE model on the same computer. Therefore the SAFE model is much more convenient to obtain the dispersion curve of the weld guided mode, and is more flexible to study different geometries and parameters.

\section{(a) Model description}

The schematic of the model is shown in Fig. 2, in which the profile has been measured from an actual welded 6 -mm-thick steel plate. Stress free conditions are imposed at the outer limit of the system. The material properties are given in Table 1, and the weld is assumed to have the same properties as the adjacent plate. In order to model the wave propagation along the weld and leaking into the side plates, an absorbing region has been attached at each side of the plate to avoid reflections from the edges (Castaings \& Lowe 2008). This region, shown in Fig. 2, has the same mass density and elastic properties as the side plate, but its viscoelasticity gradually increases with the distance away from the central axis of the system. To achieve this, the imaginary parts of its elastic moduli gradually increase according to the following law:

$$
C_{i j a}=C_{i j}^{\prime}\left[1+I \alpha_{1}\left(\frac{\left|r-r_{a}\right|}{L_{a}}\right)^{3}\right], I=\sqrt{-1}
$$

where $C_{i j}^{\prime}$ represents the stiffness of the side plate, $r_{a}$ is the distance between the inner border of the absorbing region and the central axis, $L_{a}$ is the length of the absorbing region, $r$ is the position in the absorbing region with respect to the central axis, and $C_{i j a}$ are the resulting viscoelastic moduli of the absorbing region. $\alpha_{1}$ is a coefficient that defines the proportion of the viscoelasticity at the outer limit of the absorbing region.

By introducing the imaginary part of the stiffness moduli, the propagation wave numbers, which are eigen solutions of the system, become complex $\left(k=k^{\prime}+I k^{\prime \prime}\right)$. The imaginary parts $\left(k^{\prime \prime}\right)$ represent the attenuation due to the leakage from the weld to side plates. The length of the absorbing regions has been chosen to be $800 \mathrm{~mm}$, which is twice the biggest wavelength of any radiated wave in the whole frequency range (Castaings \& Lowe 2008), and was proven to be efficient by a convergence check: when the length was increased the same solutions for the propagating modes in the waveguide was still obtained. The total width of the cross section is $2 \mathrm{~m}$, including the absorbing region. The whole geometry is meshed by 1072 triangular elements of the first order, with sidelengths comprised between 1 and $6 \mathrm{~mm}$. These elements are automatically generated by the software used, and are finer in the welded zone than in the adjacent plates. The number of degrees of freedom is 15882 .

The system is solved using the SAFE method to find values of the wave number $k$ at different frequencies. For each frequency, several solutions are obtained. For each solution, the axial component of the energy flow is calculated at each nodal position of the mesh, and the quantity is expressed by the following formula (Auld 1990):

$$
P_{x_{3}}=-\operatorname{Re}\left[\left(\frac{I \omega}{2}\right)\left(u_{1}^{*} \sigma_{31}+u_{2}^{*} \sigma_{32}+u_{3}^{*} \sigma_{33}\right)\right]
$$


Where $\sigma_{31}, \sigma_{32}$ and $\sigma_{31}$ are the axial stress components; $u_{1}^{*}$, $u_{2}^{*}$ and $u_{3}^{*}$ are the complex conjugate of the vertical, horizonal and axial displacements respectively. Solutions with higher axial component of the energy-flow in the weld cap than in the side plates generally represent modes guided along the weld and possibly radiating in the plates, while other solutions represent resonances of the whole system and are unwanted. From our calculation, several weld guided modes have been found in the low frequency range. In this paper, we will only compare and discuss two fundamental modes, one is the compression mode, which has previously been discovered experimentally (Sargent 2006) and the other a new interesting mode which has a similar mode shape as the SH0 mode in the plate.

\section{(b) Numerical results at single frequency}

The compression weld guided mode at $100 \mathrm{kHz}$ is shown in Fig. 3 with the eigenvalue $k=115.486-3.034 \times 10^{-2} i / \mathrm{m}$, from which the corresponding phase velocity is: $C_{p h}=5440.6 \mathrm{~m} / \mathrm{s}$ and the attenuation is: $\alpha=0.263 \mathrm{~dB} / \mathrm{m}$. A snapshot of the axial component of energy flow is shown in Fig. 3(a), which indicates that the energy is concentrated in and close to the weld. The mode shape of this mode in the center of the weld along $x_{2}$ is shown in Fig. 3(b). From the figure it can be seen that the mode guided along the weld is dominated by axial displacement $u_{3}$ with respect to the vertical displacement $u_{2}$ and horizontal displacement $u_{1}$, which is similar to a S0 Lamb wave in a plate. According to the Snell-Descartes' law (Auld 1990), only modes of the lateral plates having smaller phase velocities than that of the compression weld-guided mode could be radiated in the side plates. Thus the S0 mode, with its phase velocity of $5441 \mathrm{~m} / \mathrm{s}$ (Pavlakovic et al. 1997) in a 6-mm-thick plate, can not radiate, while in principle the other two fundamental modes, A0 and SH0 could. However, since this compression weld-guided mode is symmetric with respect to the mid-plane of the plates and weld, the A0 mode, which is anti-symmetric, cannot be launched. Therefore, the SH0 mode is the only mode that can be leaked into the plates at $100 \mathrm{kHz}$, which would be radiated at an angle equal to $\theta_{\text {leak }}=\sin ^{-1}(3260 / 5440.6) \approx 36.8^{\circ}$, with respect to the direction normal to the plates-weld interface. The axial displacement $u_{3}$ which dominates this compression weld guided mode in the center of the plate along $x_{1}$ is shown in Fig. 3(c). From the figure it can be seen that the axial displacement quickly decays with distance away from the center, which indicates the energy is concentrated in and around the weld. The oscillation of $u_{3}$ represents the leakage of the SH0 wave in the plate. It can be seen that the separation distance of the oscillation peaks agrees with the projection of the wavelength of the leaky SH0 wave along the $x_{1}$ direction, using $\lambda_{\text {proj }}=\lambda_{S H 0} / \cos \theta_{\text {leak }}=40.7 \mathrm{~mm}$.

The shear mode at $100 \mathrm{kHz}$ is shown in Fig. 4 with the eigenvalue $k=194.86-$ $1.3 \times 10^{-7} i / \mathrm{m}$. The corresponding phase velocity is: $C_{p h}=3224.5 \mathrm{~m} / \mathrm{s}$, and the attenuation is zero which means there is no mode leaking to the lateral plates. To our knowledge, this non-leaky mode has not been observed before, and it is very interesting for long distance inspection. A snapshot of the axial component of energy flow is shown in Fig. 4(a), which indicates that the energy is concentrated in the weld. The mode shape of this shear mode in the center of the weld along $x_{2}$ is shown in Fig. 4(b). From the figure it can be seen that the mode guided along the weld is dominated by horizontal displacement $u_{1}$ with respect to the vertical 
displacement $u_{2}$ and axial displacement $u_{3}$, which is similar to a SH0 wave in a plate. Fig. 4(c) shows the horizonal displacement $u_{1}$ in the center of the plate along $x_{1}$, which indicates that the energy is concentrated in the weld, but this mode has no leakage and thus no oscillation in the adjacent plates.

\section{(c) Dispersion curve of the weld guided modes}

The dispersion curves can be obtained by repeating the eigen calculations over a desired frequency range and the various modes identified by comparing the mode shapes.

Fig. 5 shows the phase velocity and attenuation dispersion curve of the compression weld guided mode from 50 to $500 \mathrm{kHz}$ produced by the SAFE method. The phase velocity dispersion curves of simple flat plates with $6 \mathrm{~mm}$ and $10 \mathrm{~mm}$ thickness are also plotted in figure 5(a) for comparison, confirming the similarity of the weld guided mode to the S0 Lamb mode in the plate. As discussed earlier, this compression mode leaks the SH0 wave in the side plates when the phase velocity of this mode is higher than that of the SH0 wave in the plate; however according to the Snell-Descartes' law after $410 \mathrm{kHz}$ it becomes a non-leaky mode when its phase velocity is lower than that of the SH0 wave in the plate, thus the zero attenuation after $410 \mathrm{kHz}$ in Fig. 5(b) can be expected. In the attenuation dispersion curve in Fig. 5(b), there can be seen two peaks at 200 and $350 \mathrm{kHz}$ and a dip at $250 \mathrm{kHz}$. A similar phenomenon has also been observed by Castaings and Lowe (2008), who showed that it can be explained by studying the normalized energy-flow through the weld-plate interface which shows a similar curve.

Fig. 6(a) shows the phase velocity dispersion curve of the shear welded-guided mode from 50 to $250 \mathrm{kHz}$. As is known, the $\mathrm{SH} 0$ mode in a plate is a non-dispersive mode, thus the phase velocity is constant at all frequencies, which is shown by the dashed line in the figure. From the figure it can be seen that the shear weld-guided mode has lower phase velocity than the SH0 mode in the plate. Also this mode is very much less dispersive than the compression weld-guided mode, which is another advantage for applying this mode to long range weld inspections. The imaginary part of the wavenumber of this mode stays zero at all frequencies which confirms that this mode is non-leaky.

\section{ENERGY TRAPPING EFFECT}

In order to explain the reason for the energy trapping effect of the weld guided mode, the geometry has been separated into two parts, which are the steel weld and a 6 -mm-thick steel plate. The fundamental propagation modes in these two geometries have been calculated separately.

The dispersion curves and the mode shape of the propagation modes in the weld can be calculated by the SAFE method while in the plates they can be calculated by well-established analytical methods (Lowe 1995; Pavlakovic et al. 1997). There are four fundamental modes which may exist in the weld, and the displacements in the cross section (mode shapes) at $100 \mathrm{kHz}$ are plotted in Fig. 7. It can be seen from the figure that at low frequency the torsional mode is dominated by the circumferential displacement; flexural modes 1 and 2 are dominated by the horizontal and vertical displacement respectively. The longitudinal mode is dominated by the 
axial displacement. Comparing the mode shapes of these four modes in the weld and the fundamental modes in the plate, it can be found that the longitudinal mode and flexural modes 1 and 2 have similar mode shapes as the S0, SH0 and A0 modes in the plate respectively, while the torsional mode does not have any similar modes in the plate.

The phase velocity dispersion curves of the fundamental propagation modes in the weld are shown in Fig. 8 by the solid lines, and compared with the dispersion curves of the S0, SH0 and A0 modes of a 6-mm-plate, which are shown in dashed lines. From the figure, it can be seen that the phase velocity of the longitudinal and flexural mode 1 are slower than their similar modes in the plate, which are the S0 and SH0 mode respectively. The Snell-Descartes' law (Auld 1990) would impose the condition that, when the weld and plate are joined up, the waves must have the same axial velocity in both parts. The combined axial velocity should be expected to be something between the velocity in the weld and that in the plate. Thus, if the velocity in the weld mode is less than its similar mode in the plate, the combined velocity should be less than the plate velocity. It is also known from the Snell-Descartes' law (Auld 1990), that if a wave is constrained by the boundary conditions at an interface to have a slower phase velocity than that in the adjoining medium, then it can propagate energy only along the interface, not away from it. Therefore while the combined mode, which could be the compression mode or the shear mode, is propagating along the weld, the energy of the mode will be trapped in and around the weld. The phase velocity of the flexural mode 2 is higher than its similar mode (A0 mode) in the plate, thus it cannot be trapped. Therefore we can summarize that the condition of the trapping effect should follow the rule: the propagation modes in the weld cap should have similar mode shapes as the corresponding modes in the plate, but have slower phase velocity. It should be noted that the geometry of a weld could be less regular than that which is discussed in our model and the material of a weld could be different from the adjacent plate. However, the weld guided modes can always exist as long as the phase velocity of the those modes in the weld cap are slower than their similar modes in the plate, so that the energy is trapped in the welded zone.

\section{EXPERIMENT}

\section{(a) Experimental setup}

An experimental setup was designed to validate the shear weld guided mode since it is particularly attractive for NDE. In order to achieve an accurate experimental validation with low noise, a machined $600 \mathrm{~mm}$ by $1200 \mathrm{~mm}$ aluminum plate $\left(\rho=2700 \mathrm{~kg} / \mathrm{m}^{3}, C_{l}=6474 \mathrm{~m} / \mathrm{s}, C_{s}=3051 \mathrm{~m} / \mathrm{s}\right.$, at temperature $\left.20^{\circ} \mathrm{C}\right)$ was used instead of using the actual welded plate. The plate was originally $10 \mathrm{~mm}$ thick, and was machine to be $3 \mathrm{~mm}$ thick with a $10 \mathrm{~mm}$ by $10 \mathrm{~mm}$ square bar in the center. Although it was not a welded plate, this simple idealized geometry enables the same principle of the energy trapping effect to be examined as a validation. A schematic of the experimental setup is shown in figure 9. A wide-band piezoelectric shear transducer(Panametrics V154) was attached on the top of the bar at the edge, as shown, to excite the shear mode with a 5 cycle Hanning windowed tone burst. Its orientation was such to impose its oscillation force in the horizontal direction 
as seen in this view (lateral to the bar). The signals were generated using a Wavemaker (Macro Design Ltd, UK) instrument. A laser interferometer (Polytec OFV 2700 , with dual differential fiber optic lines) was used to pick up the horizontal displacement at two monitor lines. One was along the center of the bar $200 \mathrm{~mm}$ to $500 \mathrm{~mm}$ from the source, and the other was across the bar $300 \mathrm{~mm}$ from the source, as shown in the figure. A LeCroy 9400A Storage Oscilloscope was used to store the time trace of the signal and the data was then transferred to a computer for processing.

\section{(b) Validation of group velocity and attenuation}

The group velocity $C_{g r}$ can be extracted from the measured signals by $C_{g r}=$ $\Delta S / \Delta T$ at any chosen frequency. Here $\Delta T$ is the flight time of the wave packets propagating along the bar at two different locations on monitor line one, which can be determined by calculating the shift of the Hilbert envelope of the measured signals, and $\Delta S$ is the distance between the different locations. The accuracy of the results was enhanced by measuring at several different locations and taking averages. A similar SAFE model as described in Sec.3 but with different geometry and material was developed to compare with the experimental results and the shear guided mode which was measured in the experiment was picked up. Figure 10 shows the dispersion curve of the group velocity measured in the experiment, and the theoretically predicted curve by the SAFE method, which was obtained by doing a numerical derivation $C_{g r}=d \omega / d k^{\prime}$ of the SAFE phase velocity results. From the figure we can see that the measured results agree very well with the theoretical predictions.

Figure 11 shows the displacement amplitude measured at different locations along monitor line one normalized by the displacement amplitude at $200 \mathrm{~mm}$ from the source at $120 \mathrm{kHz}$. For comparison, the beam spreading wave on a plane plate from a point source is also plotted in the figure, following the well known amplitude decay of approximately $1 / \sqrt{r}$ (Wilcox 2004). From the figure it can be seen that the measured shear weld-guided mode has slight attenuation, which might come from scattering or material damping, although theoretically the attenuation should be zero, but this is very much less than the attenuation of the beam spreading wave.

\section{(c) Validation of energy concentration effect}

In order to validate the trapping effect of the weld guided mode, a series of points on line two, shown in Fig. 9, $300 \mathrm{~mm}$ from the source, were monitored, and the maximum amplitude of displacement of these points at $120 \mathrm{kHz}$ was recorded and plotted in figure 12. From the figure it can be seen that the amplitude of displacement quickly decays with distance away from the central axis of the bar. Comparing to the displacement distribution of a beam-spreading wave from a point source on a plane plate without a weld, which is a circular crested wave (Auld 1990) and is straight in the figure because it is a long way from the source, it can be confirmed that the energy is concentrated in and around the weld region in the weld guided mode. The SAFE prediction is also plotted in the figure which shows perfect agreement with the experimental data. 


\section{CONCLUSIONS}

Feature guided waves are interesting for large area inspections, and the particular case of a compression wave in a weld has previously been investigated experimentally, and also theoretically by time step FE simulations. In this paper, the Semi Analytical Finite Element method has been applied to study the wave propagation along the weld and possibly leaking into the surrounding plates. A non-leaky and almost non-dispersive mode, named the shear weld-guided mode, has been discovered during the modal study and compared with the compression weld-guided mode observed earlier. It can be explained that the propagation mode can be trapped in the weld when it has a similar mode shape as in the side plates but with lower phase velocity. Experiments have been undertaken to validate the existence of the shear weld-guided mode and the accuracy of the FE model, showing very good agreement.

During the modal calculation, we also found some high order weld-guided modes; however for simplicity only the two fundamental weld-guided modes were discussed since our interest is to reveal the principle of feature-guiding. These higher order modes might be interesting for NDE applications at higher working frequencies, if they are more sensitive to defects. Future work will investigate the potential of using feature guided waves for inspection.

Although this study has focused on a weld geometry, it is clear from the revealed principles of the guiding effect that feature-guiding should be expected in any geometric feature whose effect is to lower the phase velocity of the waves. Therefore a similar opportunity for long distance feature-guided propagation may be possible in many other kind of structural features, such as lap joints, stiffeners, or other commonly occurring design elements.

The authors are grateful to the Engineering and Physical Science Research Council (EPSRC), and to Shell Exploration \& Production, and Nexia Solutions, for funding this work.

\section{References}

Alleyne, D. N., Pavlakovic, B., Lowe, M. J. S. \& Cawley, P. 2001 Rapid, long range inspection of chemical plant pipework using guided waves. Insight 43, 93-101.

Auld, B. A. 1990 Acoustic fields and waves in solids, vol. 1. Malabar, Florida: Krieger Publishing Company.

Castaings, M. \& Lowe, M. J. S. 2008 Finite element model for waves guided along solid systems of arbitrary section coupled to infinite solid media. J. Acoust. Soc. Am. 123, 696-708.

COMSOL 2008 User's Guide and Introduction, Version 3.4 by -COMSOL AB, http://www.comsol.com.

Gavrić, L. 1995 Computation of propagative waves in free rail using a finite element technique. J. Sound. Vib. 185,531-543.

Hayashi, T., Song, W. J. \& Rose, J. L. 2003 Guided wave dispersion curves for a bar with an arbitrary cross-section, a rod and rail example. Ultrasonics 41, 175-183.

Juluri, N., Lowe, M. J. S. \& Cawley, P. 2007 The guiding of ultrasound by a welded joint in a plate. In Review of progress in quantitative NDE (ed. D. O. Thompson \& D. E. Chimenti), pp. 1079-1086, vol. 26. New York: Plenum Press.

Lagasse, P. 1972 Dispersion of axially symmetric waves in empty and fluid-filled cylindrical shells. Acustica 27,317-329. 
Lee, B. C. \& Staszewski, W. J. 2003 Modelling of Lamb waves for damage detection in metallic structures: Part I. Wave propagation. Smart Mater. Struct. 12, 804-814.

Lowe, M. J. S. 1995 Matrix techniques for modelling ultrasonic waves in multilayered media. IEEE Trans. Ultrason. Ferroelectr. Freq. Control 42, 525-542.

Lowe, M. J. S. \& Diligent, O. 2002 Low-frequency reflection characteristics of the S0 lamb wave from a rectangular notch in a plate. J. Acoust. Soc. Am. 111, 64-74.

Orrenius, U. \& Finnveden, S. 1996 Calculation of wave propagation in rib stiffened plate structures. J. Sound Vib. 198, 203-224.

Pavlakovic, B., Lowe, M. J. S., Alleyne, D. N., \& Cawley, P. 1997 DISPERSE: A general purpose program for creating dispersion curves. In Review of progress in quantitative NDE (ed. D. O. Thompson \& D. E. Chimenti), pp. 185-192, vol. 16. New York: Plenum Press.

Postnova, J. \& Craster, R. V. 2008 Trapped modes in 3D topographically varying plates. IMA Journel of Applied Mathematics 73, 950-963.

Predoi, M. V., Castaings, M., Hosten, B. \& Bacon, C. 2007 Wave propagation along transversely periodic structures. J. Acoust. Soc. Am. 121, 1935-1944.

Rose, J. L., Avioli, M. J. \& Song, W. J. 2002 Application and potential of guided wave rail inspection. Insight 44, 353-358.

Sargent, J. P. 2006 Corrosion detection in welds and heat affected zones using ultrasonic Lamb waves. Insight 48, 160-167.

Wilcox, P., Evans, M., Lowe, M. J. S. \& Cawley, P. 2002 Dispersion and excitability of guided acoustic waves in isotropic beams with arbitrary cross-section. In Review of progress in quantitative NDE (ed. D. O. Thompson \& D. E. Chimenti), pp. 203-210, vol. 21. New York: Plenum Press.

Wilcox, P., Pavlakovic, B., Evans, M., Vine, K., Cawley, P., Lowe, M. J. S. \& Alleyne, D. N. 2003 Long range inspection of rail using guided waves. In Review of progress in quantitative NDE (ed. D. O. Thompson \& D. E. Chimenti), pp. 236-249, vol. 22. New York: Plenum Press.

Wilcox, P. 2004 Modelling the excitation of lamb and sh waves by point and line sources. In Review of progress in quantitative NDE (ed. D. O. Thompson \& D. E. Chimenti), pp. 206-213, vol. 23. New York: Plenum Press.

Wilcox, P., Lowe, M. J. S. \& Cawley, P. 2005 Omnidirectional guided wave inspection of large metallic plate structures using an emat array. IEEE Trans.Ultrason. Ferroelectr. Freq. Control 52, 653-665. 
Table 1. Mechanical properties for materials used in SAFE modelling.

\begin{tabular}{lccc} 
& Density $\left(\mathrm{kg} / \mathrm{m}^{3}\right)$ & Bulk longitudinal velocity $(\mathrm{m} / \mathrm{s})$ & Bulk shear velocity $(\mathrm{m} / \mathrm{s})$ \\
\hline Steel & 7932 & 5959 & 3260 \\
Aluminium & 2700 & 6474 & 3051 \\
\hline
\end{tabular}




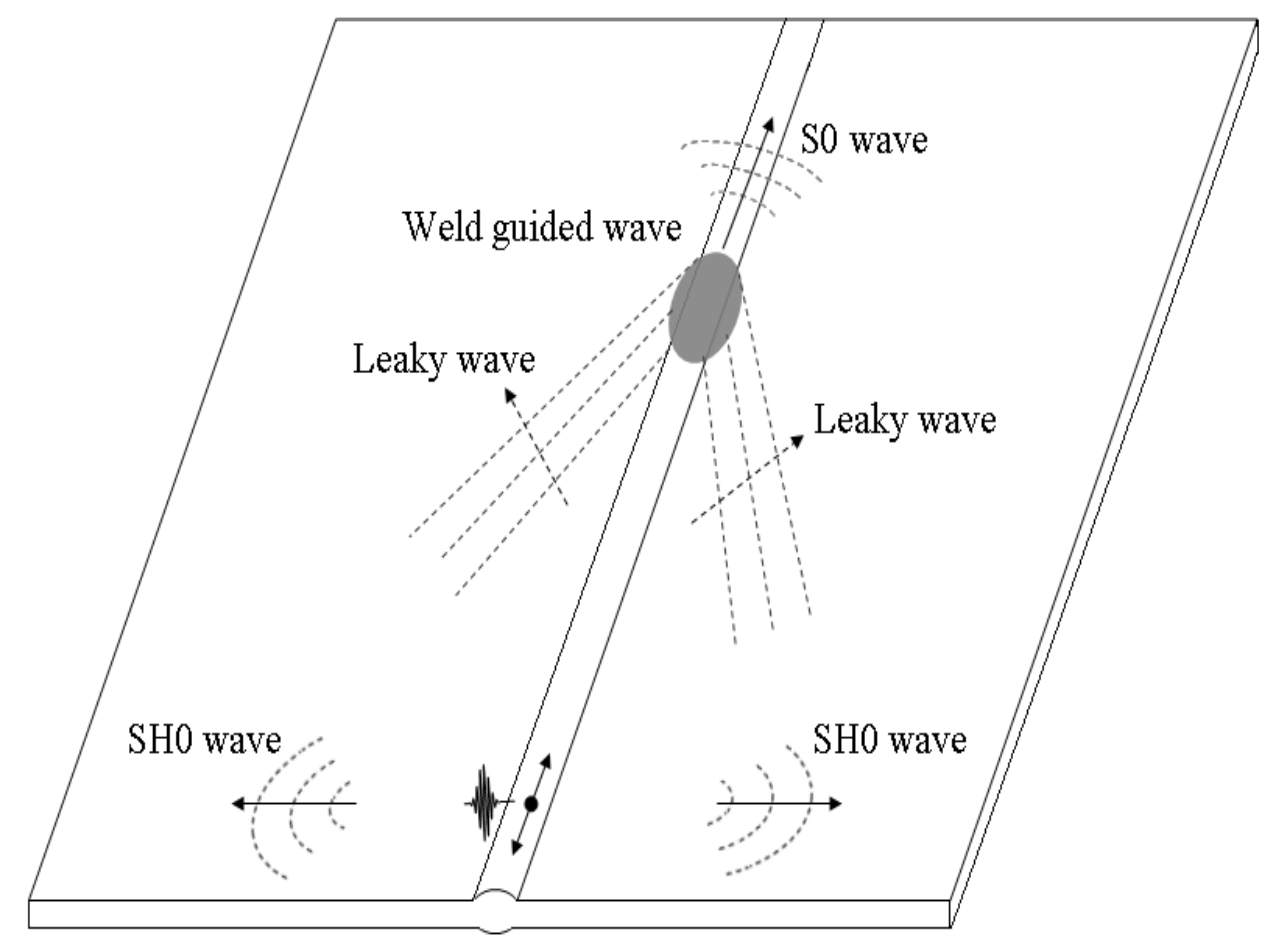

Figure 1. Schematic of the propagation of a feature-guided wave on a welded plate from a pulse excitation. 


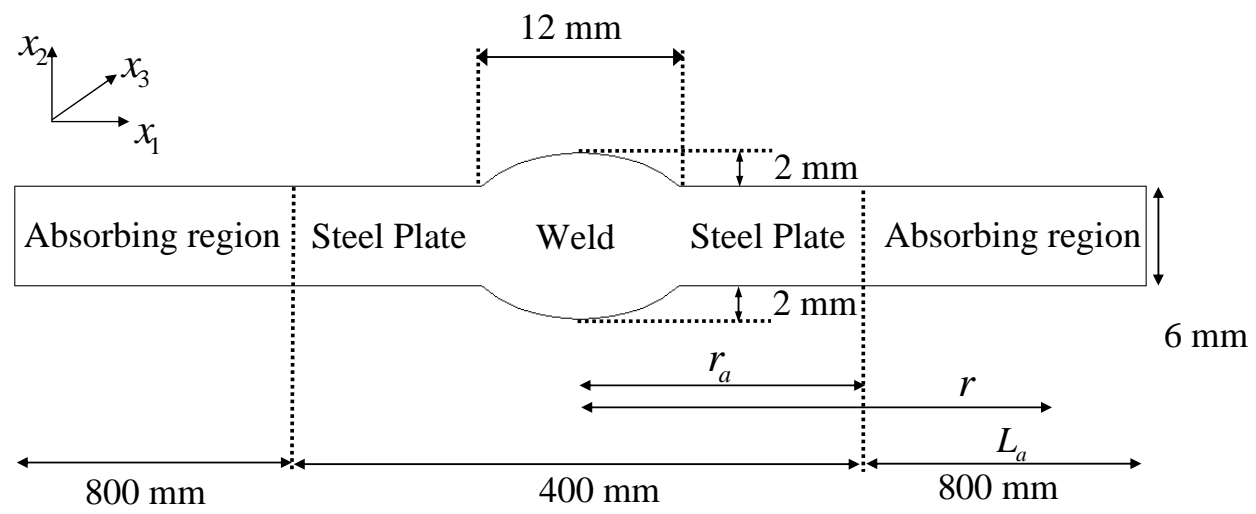

Figure 2. The schematic of the two dimensional SAFE model of a welded plate. 


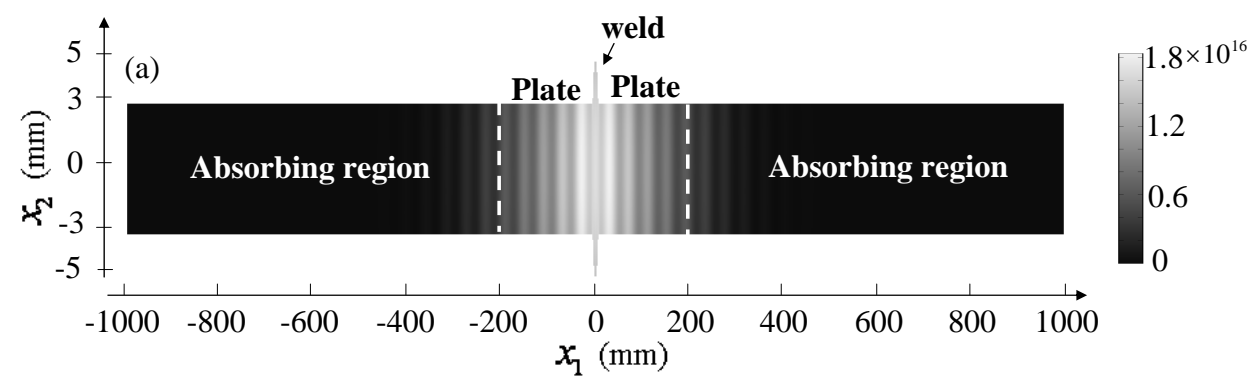

(b)

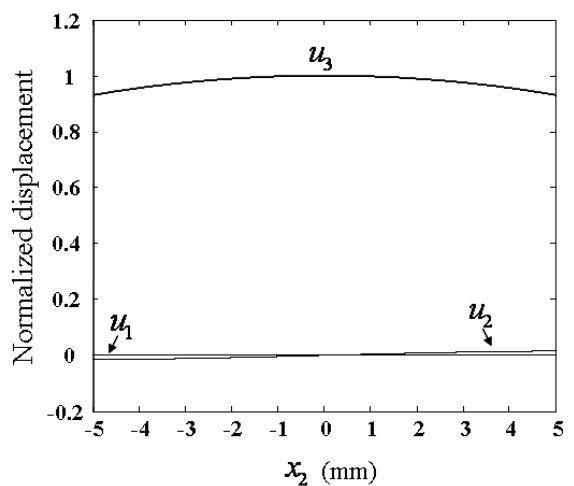

(c)

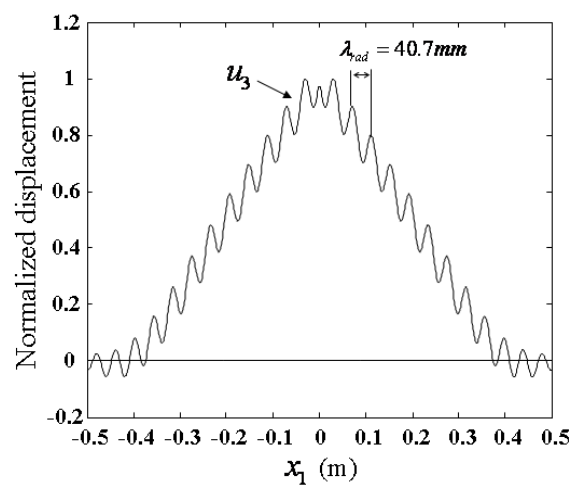

Figure 3. Compression weld guided mode at $100 \mathrm{kHz}$ : (a) snapshot of the axial component of energy-flow (white=high energy-flow, black=low energy-flow), (b) the mode shape in the center of the weld along $x 2(\mathrm{u} 1, \mathrm{u} 2, \mathrm{u} 3$ represent displacements of $\mathrm{x} 1, \mathrm{x} 2$ and $\mathrm{x} 3$ respectively) (c) the axial displacement in the center of the plate along $x 1$. 

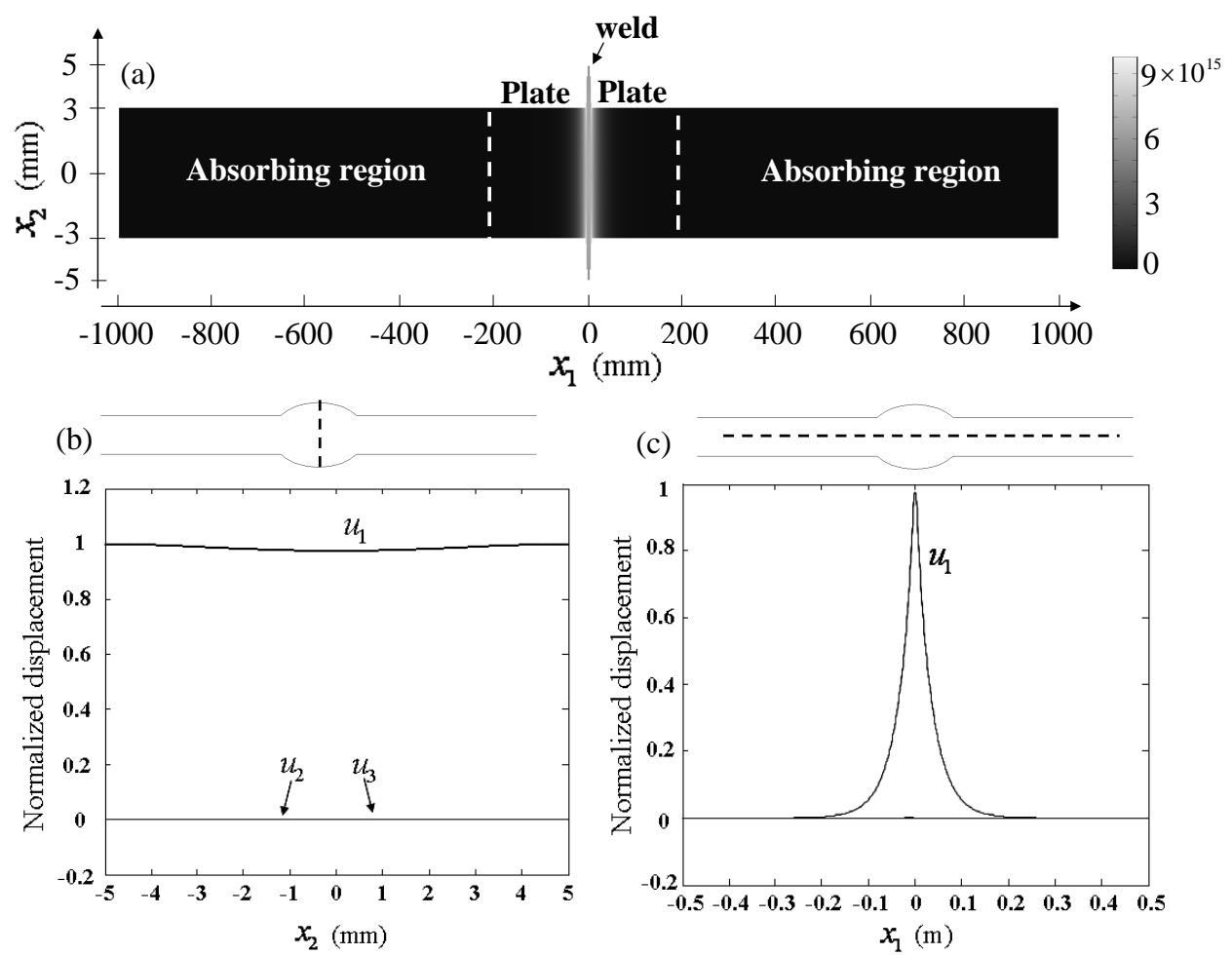

Figure 4. Shear weld guided mode at $100 \mathrm{kHz}$ : (a) snapshot of the axial component of energy-flow (white=high energy-flow, black=low energy-flow), (b) the mode shape in the center of the weld along $x 2$ ( $\mathrm{u} 1, \mathrm{u} 2$, u3 represent displacements of $\mathrm{x} 1, \mathrm{x} 2$ and $\mathrm{x} 3$ respectively) (c) the horizontal displacement in the center of the plate along $x 1$. 

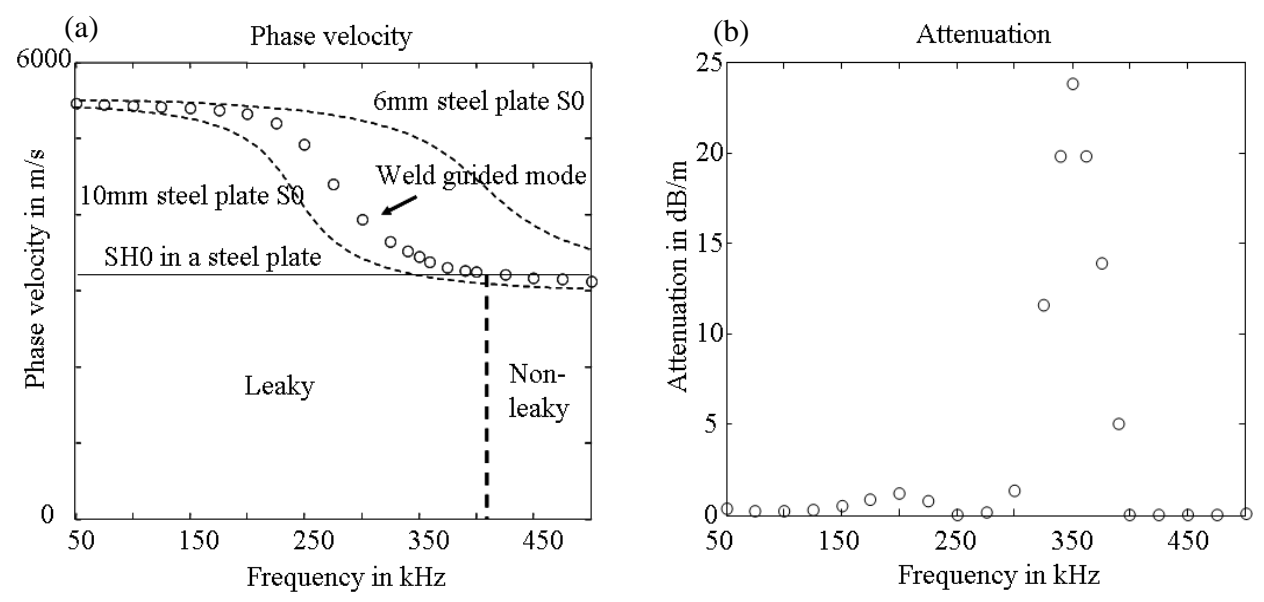

Figure 5. Phase velocity (a) and attenuation (b) dispersion curve of compression weld guided mode predicted by SAFE method. 
Phase velocity

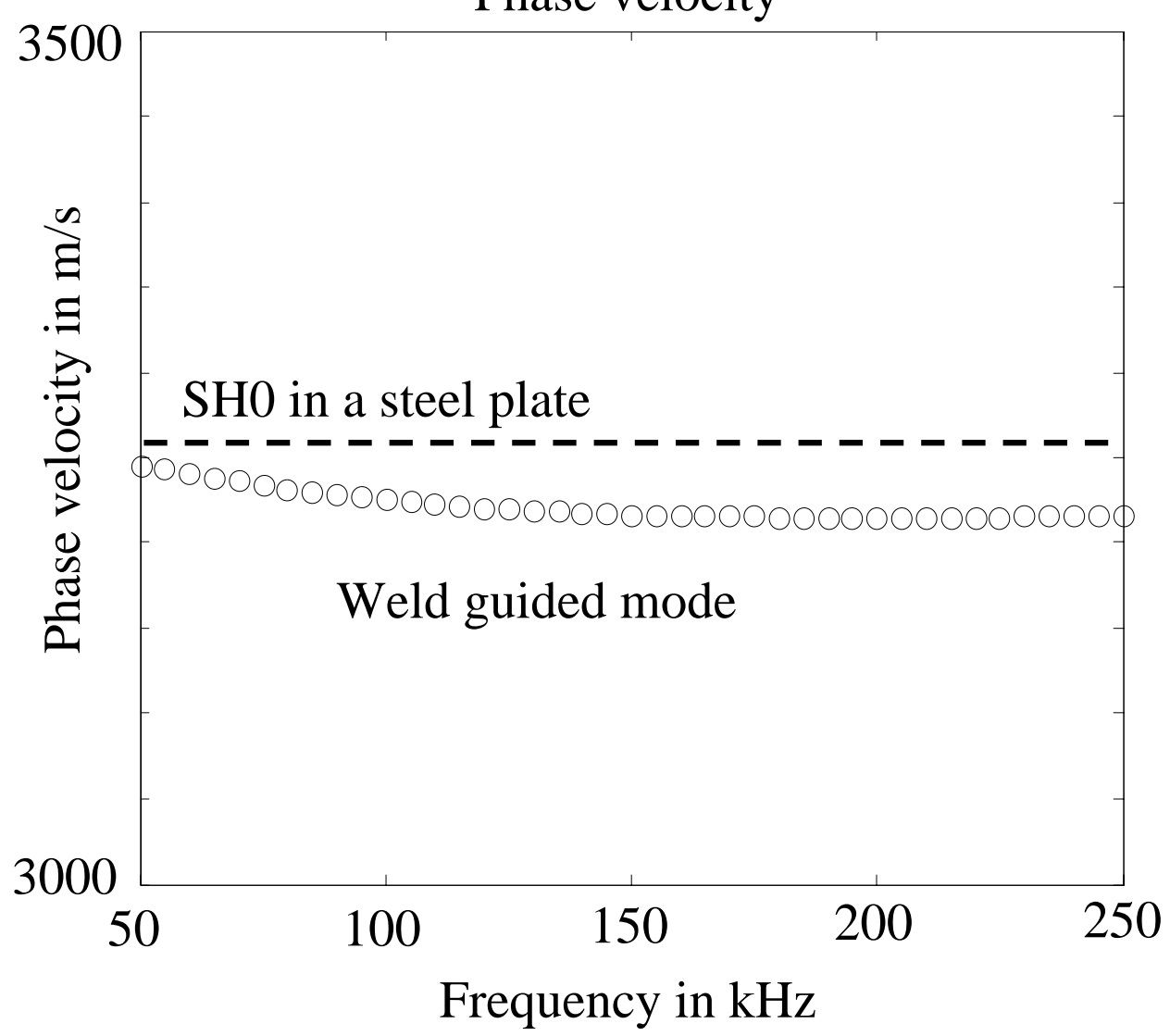

Figure 6. Phase velocity dispersion curve of shear weld guided mode predicted by SAFE method. 
Longitudinal mode

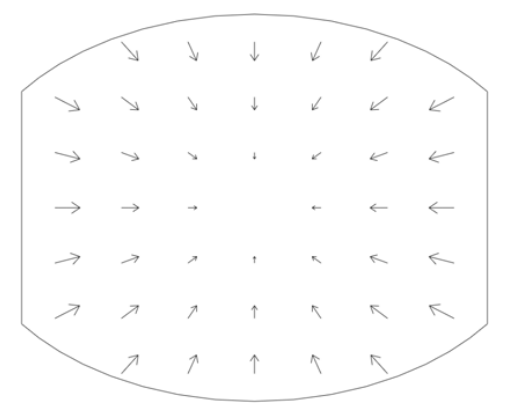

Flexural mode 1

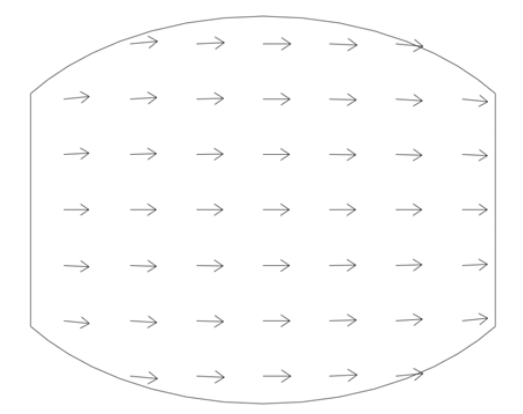

\section{Torsional mode}

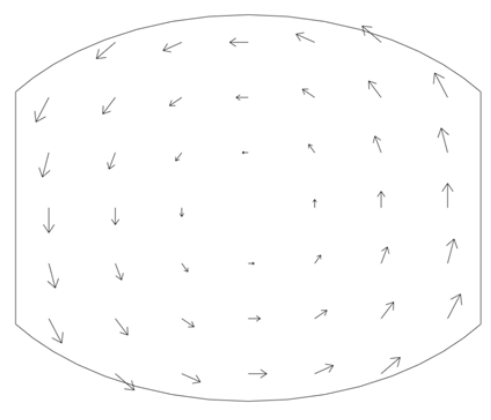

Flexural mode 2

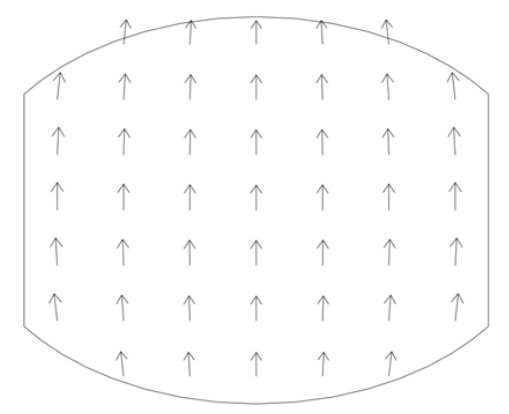

Figure 7. Mode shapes of four fundamental propagation modes of the weld at $100 \mathrm{kHz}$. Arrows indicate displacements in the cross section. 


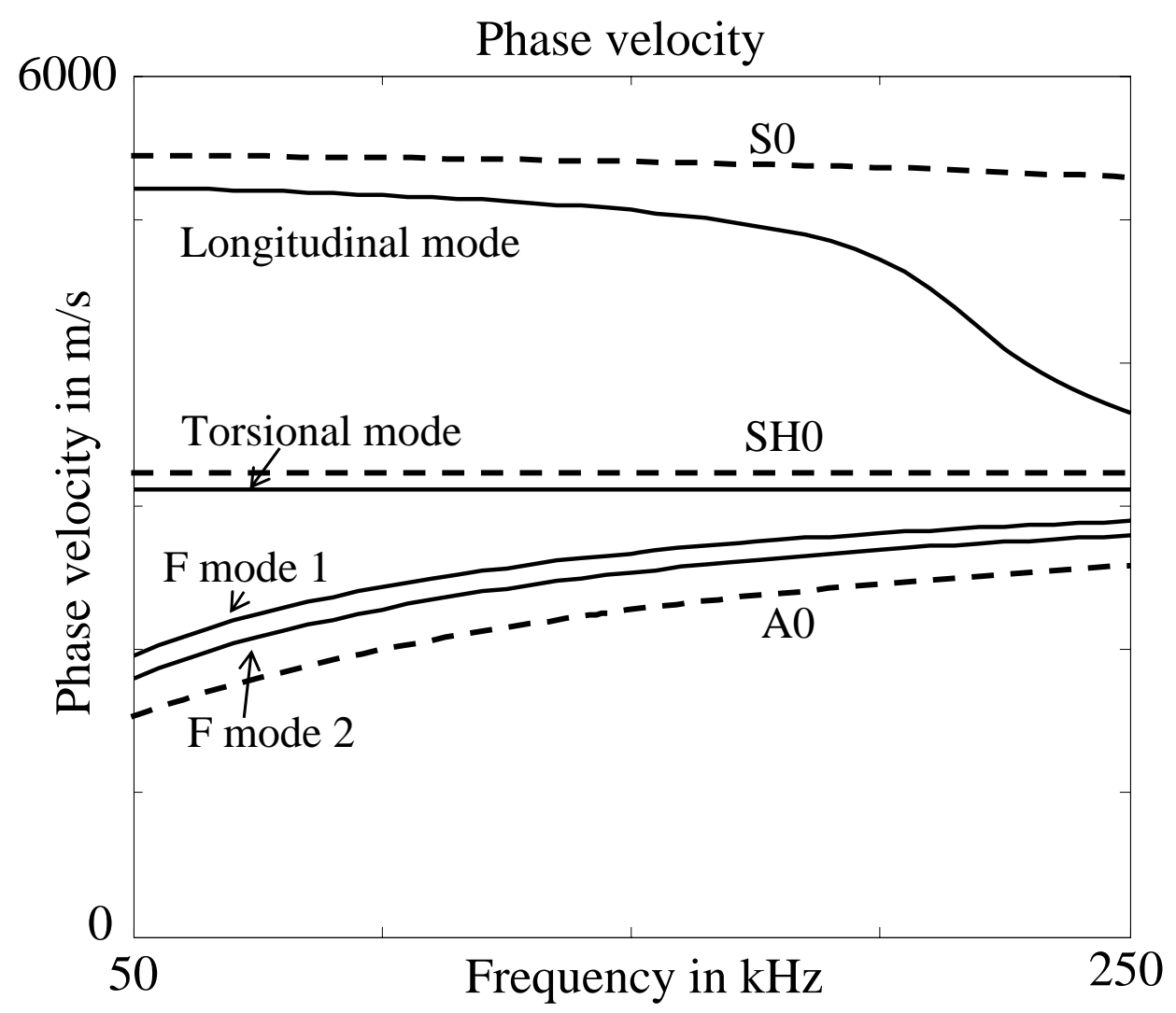

Figure 8. Phase velocity dispersion curves of the fundamental propagation modes in the steel weld and in the 6-mm-thick steel plate. 


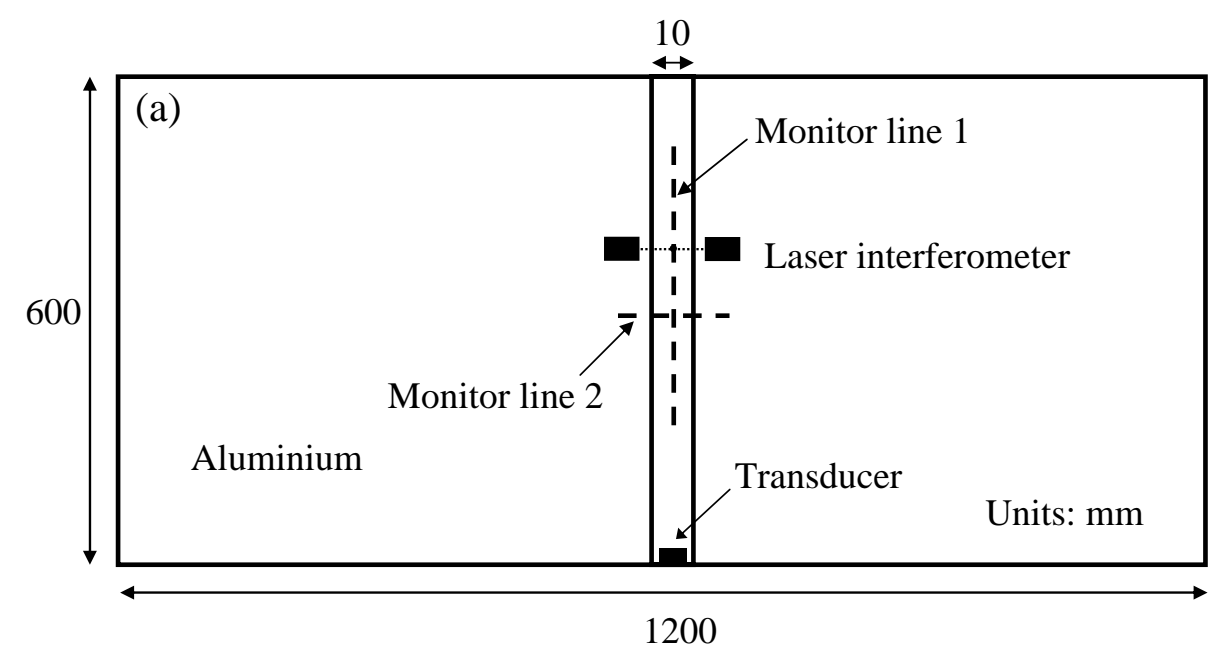

(b) Monitor line 2

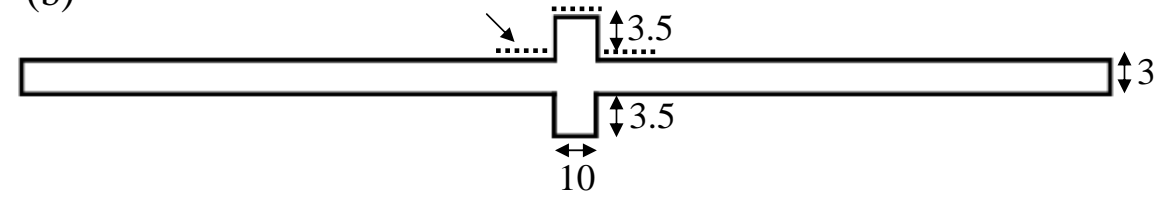

Figure 9. Experimental setup. (a) plan view, (b) side view. 


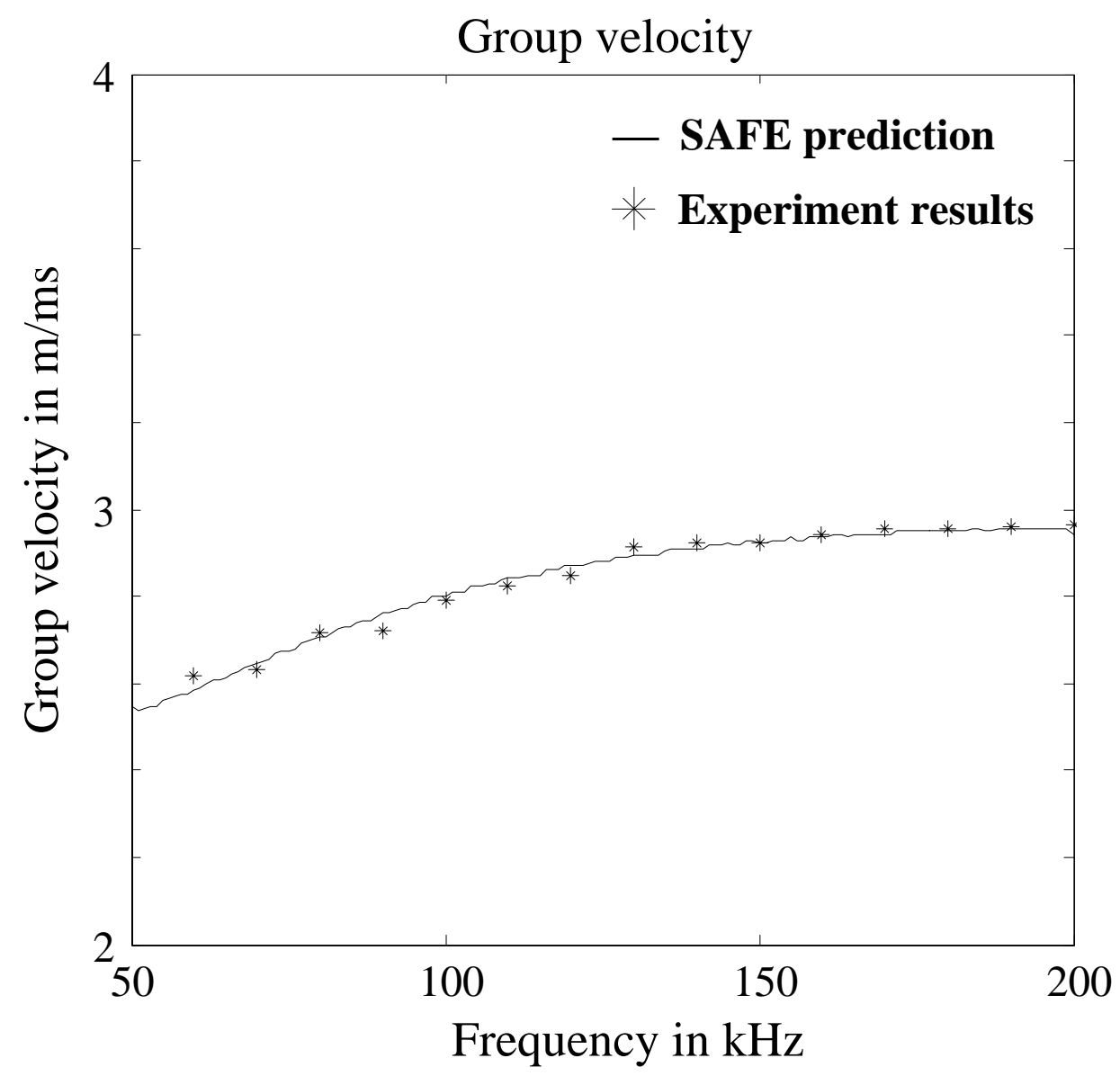

Figure 10. Measured (stars) and theoretically predicted (line) group velocity dispersion curve of the shear weld-guided mode. 


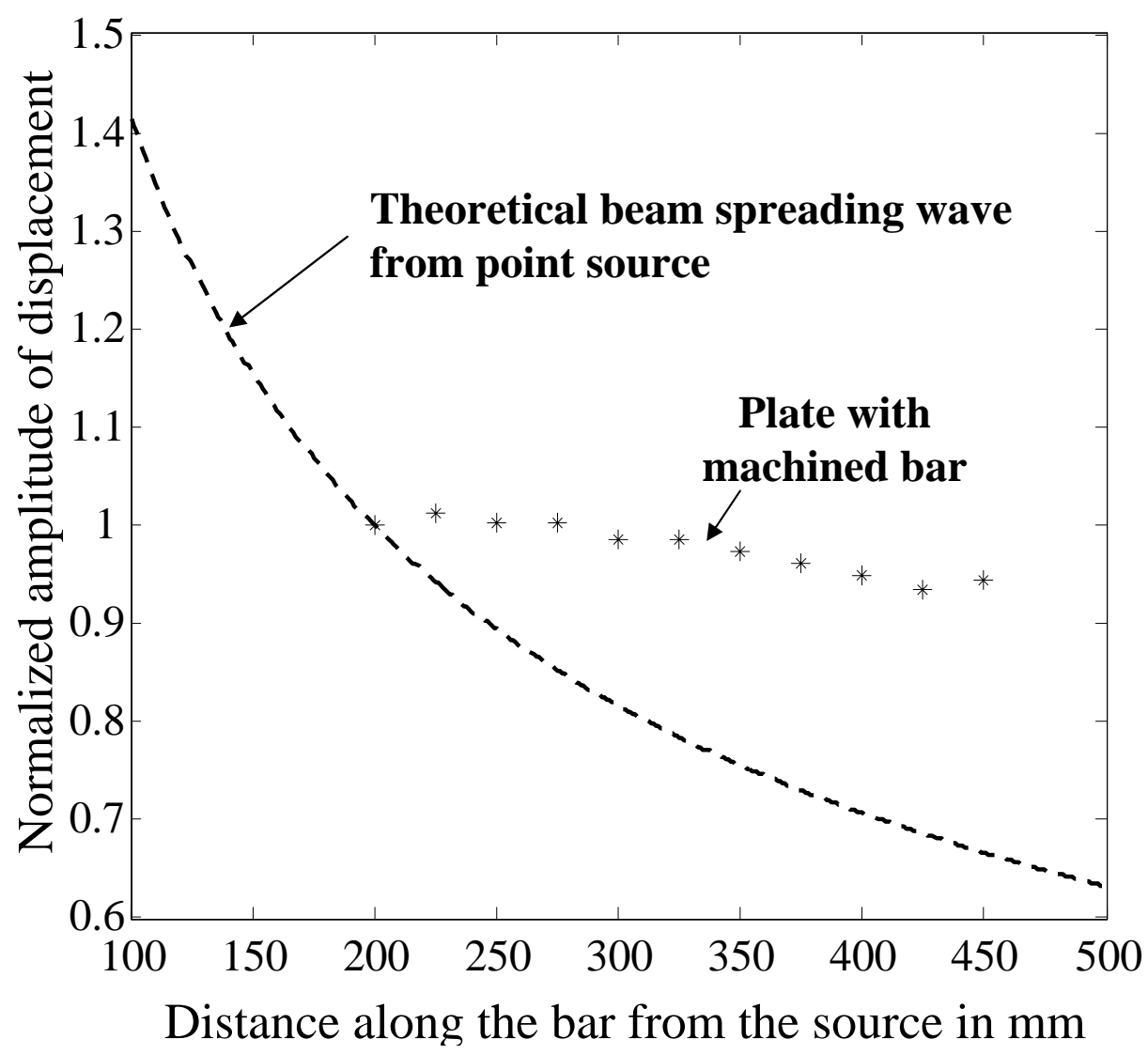

Figure 11. Normalized displacement amplitude measured at different locations along monitor line one (stars) and comparison with the beam spreading wave from a point source on a plate (dashed line). 


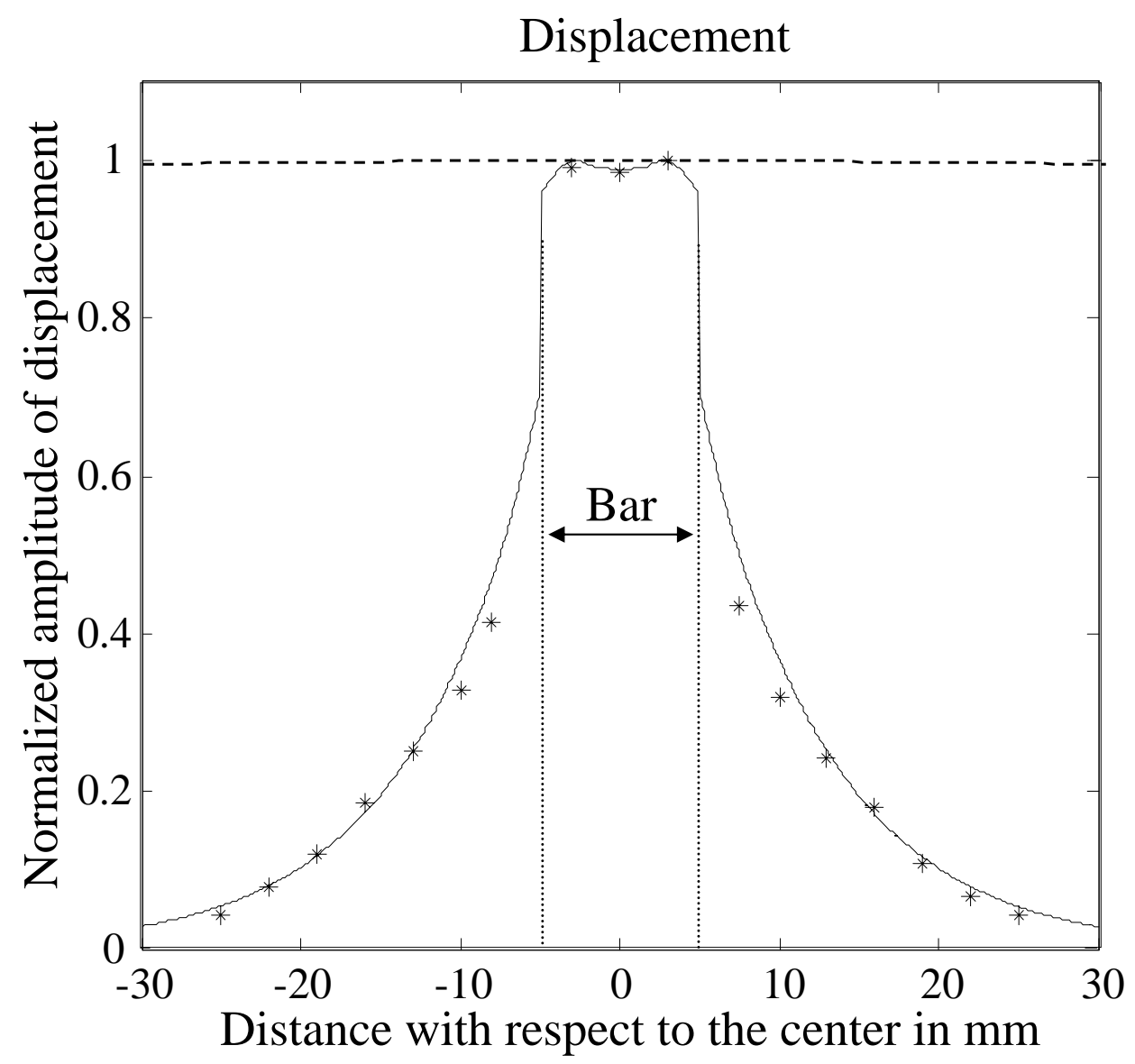

Figure 12. Measured (stars) and theoretically predicted (solid line) normalized amplitude of displacement monitored along monitor line two, $300 \mathrm{~mm}$ from the source, and comparison with the beam spreading wave from a point source on a plate (dashed line). The width of the bar is also shown 